\title{
CONTRIBUCIÓN PARA LA CARACTERIZACIÓN BIOCLIMÁTICA DEL NORTE DE PORTUGAL. LA TRANSICIÓN FLORÍSTICA ATLÁNTICO-MEDITERRÁNEA
}

\author{
Álvaro MARTINS ${ }^{1}$, Antonio L. CRESPÍ ${ }^{1 *}$, Adriano CASTRO ${ }^{1}$, Claúdia P. FERNANDES $^{1}$, \\ João ROCHA ${ }^{1}$, Carlos $\mathrm{CASTRO}^{2}$, Sonia BERNARDOS ${ }^{3}$ y Francisco AMICH $^{3}$
}

\author{
${ }^{1}$ Herbário, Jardim Botânico da Universidade de Trás-os-Montes e Alto Douro (C.E.T.A.V.), \\ apdo. 1013, 5001-801 Vila Real, Portugal. \\ ${ }^{2}$ Departamento de Fitotecnia e Engenharia Rural, \\ Universidade de Trás-os-Montes e Alto Douro, Portugal. \\ ${ }^{3}$ Departamento de Botánica, Facultad de Biología, Universidad de Salamanca, Avda. Campo Charro \\ s/n., E-37008 Salamanca, España. \\ *Autor para correspondencia: acrespi@utad.pt
}

Recibido el 6 de junio de 2007, aceptado para su publicación el 17 de enero de 2008 Publicado "on line" en junio de 2008

\begin{abstract}
RESUMEN. Contribución para la caracterización bioclimática del norte de Portugal. La transición florística atlántico-mediterránea. Este trabajo se incluye en la serie de trabajos ya iniciados por Crespí et al. (2001), con el objetivo de contribuir al conocimiento bioclimático del Norte de Portugal, a través de la información florística presente. En este sentido, y para proceder a una posible cartografia de las áreas biogeográficas naturales desde una perspectiva termopluviométrica, son ahora introducidos 39 nuevos táxones para la relación ya existente. Los táxones analizados en esta aportación son: Anthemis triumfetti f. flosculosa, Arnica montana subsp. atlantica, Aster aragonensis, Carduus bourgeanus, Carduus carpetanus, Carduus platypus subsp. platypus, Carduus platypus var. granatensis, Carduus pycnocephalus, Centaurea cyanus, Centaurea geresensi, Centaurea herminii subsp. herminii, Centaurea langeana, Centaurea rivularis, Centaurea triumfetti subsp. lingulata, Leucanthemopsis pallida subsp. flaveola, Leucanthemopsis pallida subsp. pulverulenta, Leuzea rhaponticoides, Phalacrocarpum hoffmannseggii, Santolina rosmarinifolia, Santolina semidentata, Arbutus unedo, Cistus ladanifer, Cistus laurifolius, Cistus populifolius, Cistus psilosepalus, Cistus salvifolius, Erica arborea, Erica australis, Erica ciliaris, Erica cinerea, Erica lusitanica, Erica scoparia, Erica tetralix, Erica umbellata, Halimium lasianthum subsp alyssoides, Halimium lasianthum subsp lasianthum, Halimium ocymoides, Halimium umbellatum subsp umbellatum, Halimium umbellatum subsp. viscosum. De modo a poder alcanzar los objetivos propuestos, se procede a la aplicación de una rutina programática multivariada, basada en un sistema de información geográfico (SIG), a partir del cual cada uno de los táxones estudiados es georeferenciado sobre cartografía ambiental (confeccionada y publicada por el Instituto para a Conservação da Natureza, del Ministerio del Ambiente portugués), comparándola después con las caracterizaciones fitoclimáticas de Franco (1994) y de Costa et al. (1998). Los resultados obtenidos confirman no sólo un acusado efecto gradual de transición entre el área de influencia bioclimática atlántica y la mediterránea, como además la existencia de una tendencia de comportamiento fitoclimático intermedio, no considerada hasta el momento en las caracterizaciones bioclimáticas ya publicadas.
\end{abstract}

Palabras clave. Taxonomía, distribución, SIG, caracterización bioclimática. 
ABSTRACT. Approach on the climatic characterization for Northern of Portugal. The atlanticmediterranean floristic transition. Thirty-nine specific and infraspecific taxa with different occurrences in the continental portuguese area are referred in the present work. In the light of these behaviours, the climatic and geomorphological variability of the North of Portugal has been important environmental factors to explain the floristic diversity in this part of the country. In this case, the taxa analysed are: Anthemis triumfetti f. flosculosa, Arnica montana subsp. atlantica, Aster aragonensis, Carduus bourgeanus, Carduus carpetanus, Carduus platypus subsp. platypus, Carduus platypus var. granatensis, Carduus pycnocephalus, Centaurea cyanus, Centaurea geresensi, Centaurea herminii subsp. herminii, Centaurea langeana, Centaurea rivularis, Centaurea triumfetti subsp. lingulata, Leucanthemopsis pallida subsp. flaveola, Leucanthemopsis pallida subsp. pulverulenta, Leuzea rhaponticoides, Phalacrocarpum hoffmannseggii, Santolina rosmarinifolia, Santolina semidentata, Arbutus unedo, Cistus ladanifer, Cistus laurifolius, Cistus populifolius, Cistus psilosepalus, Cistus salvifolius, Erica arborea, Erica australis, Erica ciliaris, Erica cinerea, Erica lusitanica, Erica scoparia, Erica tetralix, Erica umbellata, Halimium lasianthum subsp alyssoides, Halimium lasianthum subsp lasianthum, Halimium ocymoides, Halimium umbellatum subsp umbellatum, Halimium umbellatum subsp. viscosum. Because of the appropriate management of the floristic richness, a multivariate statistic routine is applied on a Geographical Informatic System (GIS) the chorological, geomorphological and climatic characterization for each one are described in this paper. The geological substrate and the altitudinal classes are focused for the geomorphological approach, as well as two biogeographic classifications are used to correlate the occurrence of the taxa and their bioclimatic preferences. In the light of this analytical scheme, every taxa is geo-referenced upon an environmental cartography (elaborated by the Instituto para a Conservação da Natureza), compared with the Franco's (1994) and Costa's et al. (1998) phytoclimatic characterizations. The results obtained are pointing out the floristic progressive differentiation between the atlantic bioclimatic influence and the mediterranean one, as well as the existence of an intermediate bioclimatic tendency which had never been considered so far.

Key words. Taxonomy, distribution, GIS, environmental characterization.

\section{INTRODUCCIÓN}

El Norte de Portugal constituye una de las zonas de transición bioclimática (Ribeiro, 1991) y, consecuentemente, florística más notables de la Península Ibérica, encerrando la transición occidental ibérica desde el Sur de Galicia (Carballeira, 1985; Retuerto \& Carballeira, 1991). Esta circunstancia está directamente relacionada con la accidentada orografía del terreno, además de otras circunstancias ambientales, tales como la compleja variación geológica existente en esta zona (Taborda, 1987) o la irregular distribución de la población. La combinación de estas circunstancias ambientales ha provocado alteraciones significativas en la presencia y distribución de muchos táxones. Por esta razón, y continuando con la série de contribuciones florísticas iniciadas a partir de Crespí et al. (1999), son de nuevo analizados táxones que ayudan a comprender mejor el comportamiento de la flora en relación a la diversificada y compleja combinación de variables ambientales, decisivos para la riqueza florística presente en esta parte del país. En este caso son seleccionados, una vez más, táxones con distribuciones diversas (todos ellos restringidos a determinadas zonas dentro del área de estudio), teniendo en consideración el hecho de que sus distribuciones los hagan estar más correlacionados con variables ambientales (Rozeira, A. 1944; Mendonca \& Vasconcellos, 1954; Franco, 1994; Costa et al., 1998).

Como ya fue comentado en contribuciones anteriores (Costa et al., 1998; Martins et al., 2004), el carácter perpendicular de las sierras, en relación con el principal canal de desagüe protagonizado por el propio río Duero provoca una 


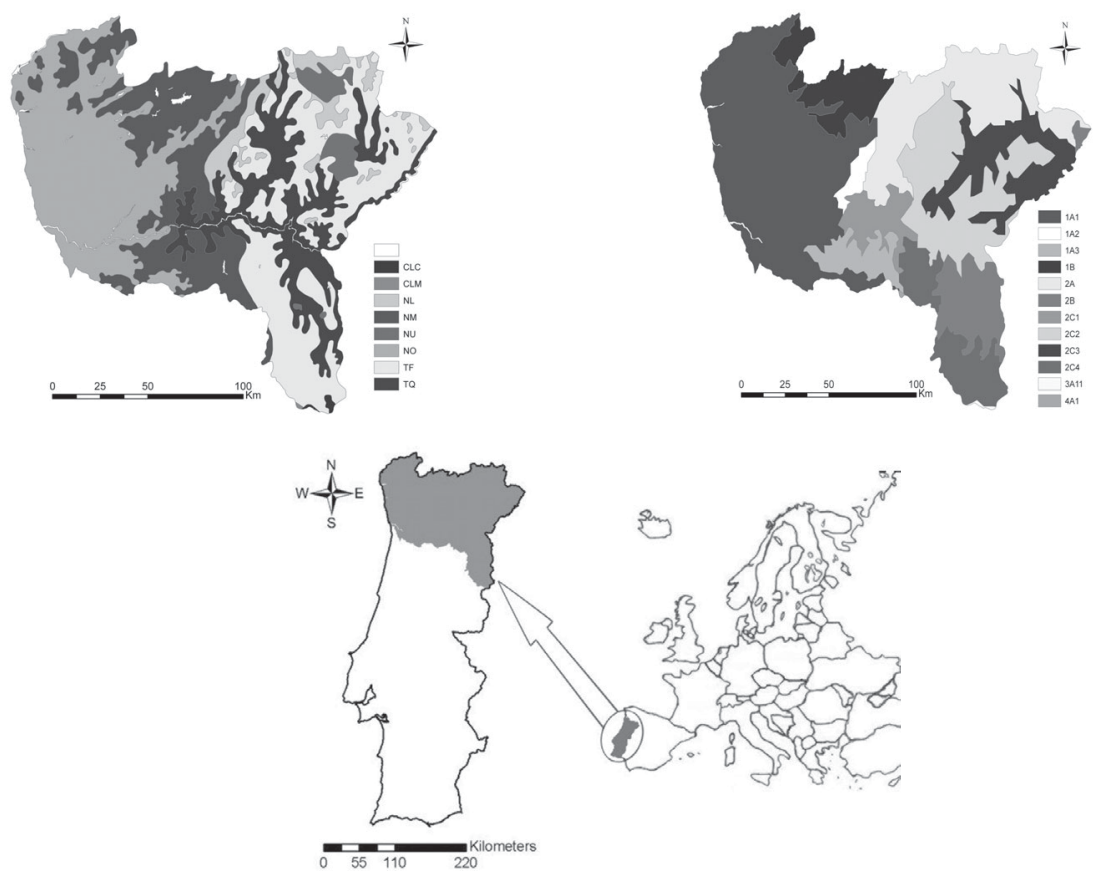

Figura 1. Caracterización de las áreas biogeográficas (a) según Franco et al. (1994) y (b) Costa et al. (1998) para el Norte de Portugal; localización del área de estudio (c). Characterization of the phytoclimatic areas according to (a) Franco et al. (1994) and (b) Costa et al. (1998); location of the study area (c).

acentuada, aunque progresiva, deshumidificación de la atmósfera. Tal fenómeno acaba por tener repercusiones directas en la variación térmica entre el interior y la franja litoral, pues la menor humedad de la primera área en relación a la segunda conlleva a una continentalización, mucho más acusada en las regiones fronterizas con Castilla-León. Al mismo tiempo, este fenómeno está muy lejos de acusar una linearidad en su comportamiento, pues el abundante afloramiento granítico impide una erosión generalizada $y$, en ocasiones abrupta, provocando la formación de valles encajados con orientaciones, en muchos casos, casi totalmente perpendiculares al río Duero. No obstante, una lectura más detallada de las contribuciones climatológicas realizadas hasta la fecha, para Portugal continental, son determinantes en lo que respecta a la distinción entre el dominio atlántico y el mediterráneo. Desde Alburquerque (1941, 1943) hasta Costa et al. (1998), pasando por Molina et al. (1992) o por Gonçalves (1985), resulta evidente el efecto de la cadena montañosa que se extiende des la Larouco (en el noroeste de la provincia de Tras-osMontes) hasta las sierras de Lapa y de Leomil (en la confluencia con la ciudad de Guarda). No obstante, los datos florísticos recogidos hasta el momento no coinciden con estas caracterizaciones bioclimáticas, pues las distribuciones de táxones típicamente atlánticos o mediterráneos encuentran en el Norte de Portugal una clara sobreposición (Crespí et al., 2005a, b).

En este sentido, las contribuciones 
fitoclimáticas más recientes, de la autoría de Franco (1994) y de Costa et al. (1998), para Portugal continental, permiten observar algunas discrepancias en relación a la frontera atlántico-mediterránea. En la primera de estas caracterizaciones, el autor estima que la influencia atlántica se extiende a lo largo del valle del río Támega, llegando a las confluencias del centro-norte portugués (hasta la vega de la ciudad de Chaves). Ya Costa et al. (op. cit.) no consideran que esta influencia deba alargarse tanto, bloqueándola a través del subsector Margado-Sanabriense (2A). Al mismo tiempo, estos mismos autores diversifican más la cordillera montañosa Gerês-Montemuro, que separa el este (constituido por las provincias de Trasos-Montes, juntamente con el Alto Duero portugués) del oeste (Miño y Duero Litoral). Estos resultados acabarían más tarde por ser respaldados en la cartografía de vegetación que, sobre Portugal continental, publicaron Capelo et al. (2007). Una representación de las caracterizaciones fitoclimáticas de Franco (op. cit.) y de Costa et al. (op. cit.), indicando además la extensión del área analizada, está presente en la figura1.

Con la presente contribución se intenta profundizar más en el comportamiento florístico-bioclimático, haciendo uso para ello de Sistemas de Información Geográfica (SIG) y de un análisis multivariante asociado al mismo.

\section{MATERIAL Y MÉTODOS}

Para desarrollar este sistema de caracterización florístico-bioclimática fueron escogidos táxones con distribuciones restringidas, esto es, endemismos ibéricos del Oeste o de la mitad Norte peninsular, así como otros con corologías más alargadas, teniendo en consideración que todos ellos estuviesen presentes en el Norte de Portugal continental. Con esta finalidad se consultaron los ejemplares de los siguientes herbarios: COI (herbario de la Universidad de Coimbra), PO (herbario de la Universidad de Porto), HVR (herbario de la Universidad de Vila Real), BRESA (herbario de la Escuela Politécnica de Braganza), LISE (Herbario de la Estación Agronómica Nacional de Oeiras), LISI (Herbario del Instituto Superior de Agronomia de la Universidad Técnica de Lisboa) y LISU (Herbario de la Facultad de Ciencias de la Universidad de Lisboa). En relación a los táxones utilizados para este análisis, fueron seleccionados un total de seis procedentes de contribuciones anteriores (Martins et al. 2004, 2006) -atendiendo a su aparente carácter indicador termopluviométrico-, procediéndose a un aumento de esta lista con los 39 siguientes (muchos de los cuales con distribuciones restringidas, dentro del área de estudio, o, también el algunos casos, limitadas a determinadas áreas geográficas del norte portugués): Anthemis triumfetti (L.) DC. f. flosculosa (Briq. \& Cavill.) R. Fernandes, Arnica montana L. subsp. atlantica A. Bolós, Aster aragonensis Asso, Carduus bourgeanus Boiss \& Reut., Carduus carpetanus Boiss. et Reuter, Carduus platypus var. granatensis (Wk.) P. Coutinho, Carduus platypus Lange subsp. platypus, Carduus pycnocephalus L., Centaurea cyanus L., Centaurea geresensis J. Arènes, Centaurea herminii Rouy subsp. herminii, Centaurea langeana Wk., Centaurea rivularis Brot., Centaurea triumfetti All. subsp. lingulata (Lag.) Vicioso, Leucanthemopsis pallida (Miller) Heywood subsp. flaveola (Hoffmanns. \& Link) Ladero \& Velasco, Leucanthemopsis pallida (Miller) Heywood subsp. pulverulenta (Lag.) Bolós et Vigo, Leuzea rhaponticoides Graells, Phalacrocarpum hoffmannseggii (Samp.) Laínz, Santolina rosmarinifolia L., Santolina semidentata Hoff \& Liink, Arbutus unedo L., 


\begin{tabular}{cccccc}
\hline Código & Variable & Código & Variable & Código & Variable \\
\hline P1 & $>2800 \mathrm{~mm}$ & $\mathrm{t} 1$ & $7,5-10,0^{\circ} \mathrm{C}$ & $\mathrm{A} 1$ & $>1300 \mathrm{~m}$ \\
P2 & $2400-2800 \mathrm{~mm}$ & $\mathrm{t} 2$ & $10,0-12,5^{\circ} \mathrm{C}$ & $\mathrm{A} 2$ & $1100-1300 \mathrm{~m}$ \\
P3 & $2000-2400 \mathrm{~mm}$ & $\mathrm{t} 3$ & $12,5-15,0^{\circ} \mathrm{C}$ & $\mathrm{A} 3$ & $900-1100 \mathrm{~m}$ \\
P4 & $1600-2000 \mathrm{~mm}$ & $\mathrm{t} 4$ & $15,0-16,0^{\circ} \mathrm{C}$ & $\mathrm{A} 4$ & $700-900 \mathrm{~m}$ \\
P5 & $1400-1600 \mathrm{~mm}$ & $\mathrm{E} 1$ & $>800 \mathrm{~mm}$ & $\mathrm{~A} 5$ & $500-700 \mathrm{~m}$ \\
P6 & $1200-1400 \mathrm{~mm}$ & $\mathrm{E} 2$ & $700-800 \mathrm{~mm}$ & $\mathrm{~A} 6$ & $400-500 \mathrm{~m}$ \\
P7 & $1000-1200 \mathrm{~mm}$ & $\mathrm{E} 3$ & $600-700 \mathrm{~mm}$ & $\mathrm{~A} 7$ & $300-400 \mathrm{~m}$ \\
P8 & $800-1000 \mathrm{~mm}$ & $\mathrm{E} 4$ & $500-600 \mathrm{~mm}$ & $\mathrm{~A} 8$ & $200-300 \mathrm{~m}$ \\
P9 & $700-800 \mathrm{~mm}$ & $\mathrm{E} 5$ & $450-500 \mathrm{~mm}$ & $\mathrm{~A} 9$ & $100-200 \mathrm{~m}$ \\
P10 & $600-700 \mathrm{~mm}$ & $\mathrm{E} 6$ & $400-450 \mathrm{~mm}$ & $\mathrm{~A} 10$ & $<100 \mathrm{~m}$ \\
P11 & $500-600 \mathrm{~mm}$ & $\mathrm{E} 7$ & $<400 \mathrm{~mm}$ & & \\
P12 & $400-500 \mathrm{~mm}$ & & & & \\
P13 & $<400 \mathrm{~mm}$ & & & & \\
\hline
\end{tabular}

Tabla 1. Relación de las variables ambientales empleadas y de los códigos respectivos (P, precipitación media; t, temperatura media; E, evapotranspiración potencial media; A, altitud). Environmental variables and respective intervals used in the present analysis ( $P$, average precipitation; $t$, average temperature; E, average potential evapotranspiration; A, altitude).

Cistus ladanifer L., Cistus laurifolius L., Cistus populifolius L., Cistus psilosepalus Sweet, Cistus salvifolius L., Erica arborea L., Erica australis L., Erica ciliaris L., Erica cinerea L., Erica lusitanica Rud., Erica scoparia L., Erica tetralix L., Erica umbellata L., Halimium lasianthum subsp alyssoides (Lam.) Greuter, Halimium lasianthum subsp. lasianthum, Halimium ocymoides (Lam.) Wk., Halimium umbellatum (L.) Spach subsp. umbellatum, Halimium umbellatum (L.) Spach subsp. viscosum (Wk.) O. Bolòs et J. Vigo. Atendiendo a las distribuciones de estos taxa, los porcentajes de sus distribuciones son los siguientes: $\quad 13 \%$ atlánticos, $28 \%$ mediterráneos, $10 \%$ euroasiáticos y los restantes $49 \%$ endémicos o subendémmicos ibéricos (incluyendo en algunos casos el SW de Francia o el NW de Marruecos). Al mismo tiempo, y teniendo presente la importancia concentrada sobre las cadenas montañosas como diferenciales climáticos de esta parte del país (especialmente el eje GerêsLarouco-Barroso-Alvão-Marão-Montemuro-
Leomil-Lapa, como frontera atlánticomediterránea clásica), los táxones escogidos presentan un 55\% de sus presencias a partir de $\operatorname{los} 500 \mathrm{~m}$.

Después de la obligatoria revisión de los ejemplares, con el objetivo de confirmar las identificaciones ya existentes, se procedió a la georreferenciación de cada uno sobre tres bases cartográficas: el mapa fitoclimático de Franco (1994), el propuesto por Costa et al. (1998) y, finalmente, la base cartográfica ambiental publicada por el Instituto do Ambiente (IA) portugués (http:// www.iambiente.pt/atlas/est/index.jsp). Sobre un soporte ArchView (ArchGis), fueron elaboradas las matrices ambientales referentes a cada una de estas georreferenciaciones: las de los mapas fitoclimáticos a partir de las áreas determinadas por los autores; las relativas a los mapas climáticos del IA portugués con parámetros relacionados con los intervalos altitudinales, de temperatura media, precipitación media y evapotranspiración potencial. Para esta última matriz se 

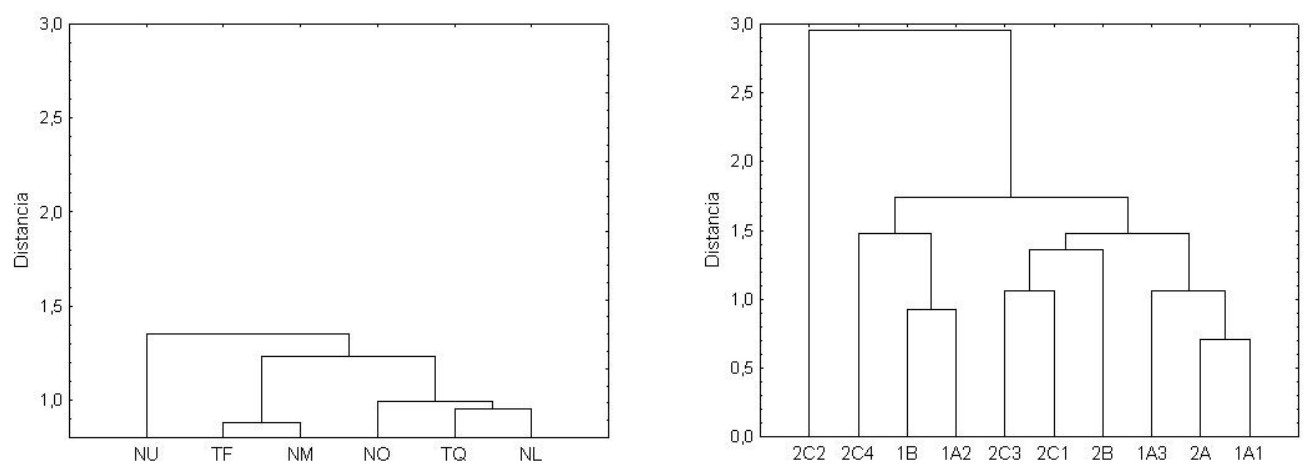

Figura 2. Dendrogramas obtenidos a partir de los valores estandarizados de la matriz de presencias medias, para la totalidad de los táxones analizados, organizados de acuerdo con a) la caracterización fitoclimática de Franco (1994), y b) con la caracterización de Costa et al. (1998) (NU, Nordeste Ultrabásico; TF, Terra Fria; NM, Nordeste Montañés; NO, Nordeste Occidental; TQ, Terra Quente; NL, Nordeste Leonês; 2C2, superdistrito Terra Quente; 2C4, superdistrito Altibeirense; 1B, subsector Geresiano-Queixense; 1A2, superdistrito Alvão-Marão; 2C3, superdistrito de Miranda-Bornes-Ansiães; 2C1, superdistrito duriense; 2B, sector Salamantino; 1A3; superdistrito Beiraduriense; 2A, subsector Margato-Sanabriense; 1A1, superdistrito Miniense-Litoral). Dendrograms elaborated from the standardized matrixes for the georeferentiation of taxa upon the phytoclimatic characterizations of a) Franco (1994) and b) Costa et al. (1998).

seleccionaron un total de 34 variables, todas ellas expuestas en la tabla 1, manteniendo el criterio establecido por Allúe Andrade (1990), intentando evitar nuevamente la redundancia entre variables y la utilización de posibles índices que pudiesen relacionarlas.

El método aplicado para este trabajo es el resultado de un desarrollo de las metodologías utilizadas por Crespí et al. (2001) y Martins et al. (2004, 2006). De este modo, y después de los sucesivos perfeccionamientos sobre este método analítico, se presenta aquí un sistema de caracterización bioclimático sustentado sobre información florística, previamente georreferenciada, que permite la representación cartográfica de correlaciones florístico-bioclimatológicas. Uno de los aspectos más relevantes de este método consiste en la capacidad de ir obteniendo grupos bioclimáticos discriminantes, de una forma gradual, de modo que sobre cada uno de los grupo inicialmente deducidos se puedan ir obteniendo subgrupos de forma sucesiva. Para tal fin, y de manera a impedir la creación de grupos estadísticamente irrelevantes, se combina cada una de estas subdivisiones con análisis discriminantes, que ayudan a detectar el grado de relevancia numérica para los subgrupos deducidos.

De este modo, fue creada una matriz numérica básica en la que cada taxon era caracterizado de acuerdo con las variables ambientales referidas, teniendo en cuenta la distribución del mismo. Sobre esta matriz se procedió a la sustitución del valor de presencia del taxon, variable a variable, por su respectivo valor medio de presencia, para, de este modo, obtener una matriz con valores contínuos. Finalmente, en esta matriz contínua se realizaba una estandarización, obteniendo un sistema comparativo equiparable por taxon (siguiendo así el proceso metodológico empleado en Martins et al. 2004, 2006).

El análisis multivariante realizado sobre 


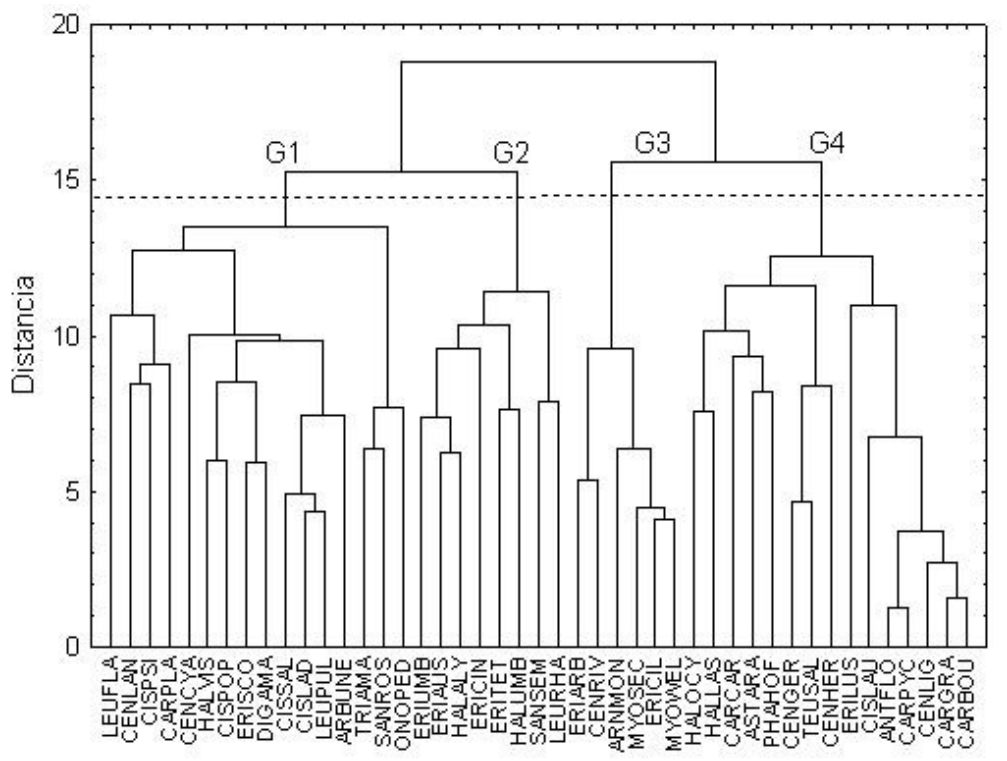

Figura 3. Representación en un dendrograma del análisis de semejanza realizado sobre la matriz de correlación calculada a partir de la matriz numérica estandarizada, las líneas fenogramáticas (en trazo discontínuo) indican los grupos obtenidos para los valores de distancias más discriminantes -G1, G2, G3 y G4-. Dendrogramatic representation for the similarity analysis from the correlation matrix of the standardized numerical matrix, the groups obtained for the most discriminant distances -G1, G2, G3 and G4- are deduced from the phenogramatic line (ANTFLO, Anthemis triumfetti f. flosculosa; ARBUNE, Arbutus unedo; ARNMON, Arnica montana subsp. atlantica; ASTARA, Aster aragonensis; CARBOU, Carduus bourgeanus; CARCAR, C. carpetanus; CARPLA, C. platypus subsp. platypus; CARGRA, C. platypus var. granatensis; CARPYC, C. pycnocephalus; CENCYA, Centaurea cyanus; CENGER, C. geresensi; CENHER, C. herminii subsp. herminii; CENLAN, C. langeana; CENRIV, C. rivularis; CENLIG, C. triumfetti subsp. lingulata; LEUFLA, Leucanthemopsis pallida subsp. flaveola; LEUPUL, L. pallida subsp. pulverulenta; LEURHA, Leuzea rhaponticoides; PHAHOF, Phalacrocarpum hoffmannseggii; SANROS, Santolina rosmarinifolia; SANSEM, S. semidentata, Arbutus unedo; CISLAD, Cistus ladanifer; CISLAU, C. laurifolius; CISPOP, C. populifolius; CISPSI, C. psilosepalus; CISSAL, C. salvifolius; ERIARB, Erica arborea; ERIAUS, E. Australis; ERICIL, E. ciliaris; ERICIN, E. cinerea; ERILUS, E. lusitanica; ERISCO, E. scoparia; ERITET, E. tetralix; ERIUMB, E. umbelata; HALALY, Halimium lasianthum subsp alyssoides; HALLAS, $H$. lasianthum subsp lasianthum; HALOCY, $H$. ocymoides; HALUMB, $H$. umbellatum subsp umbellatum; HALVIS, $H$. umbellatum subsp. viscosum; DIGAMA, Digitalis amandiana; TRIAMA, Trigonella amandiana; ONOPED, Ononis viscosa $\mathrm{ssp}$ pedroi; MYOSEC, Myosotis secunda; MYOWEL, Myosotis welwistchi; TEUSAL, Teucrium salviastrum).

esta matriz se sustentó en un estudio de semejanzas, a través de un cluster y de una determinación de grupos de comportamiento, con ayuda de un análisis canónico discriminante (CDA) -forward stepwise-. Para este último análisis clasificativo se utilizaron los estadísticos Lambda de Willks y el valor exacto de F (Gotelli \& Ellison,
2004), con el propósito de poder detectar la distancia más discriminante en cada cluster. A partir de esta rutina estadística se elabora una caracterización ambiental de cada taxon, de forma a que puedan ser establecidos los intervalos ecológicos por taxon, teniendo como base las variables ambientales seleccionadas para la georreferenciación. 
$\mathrm{a}$
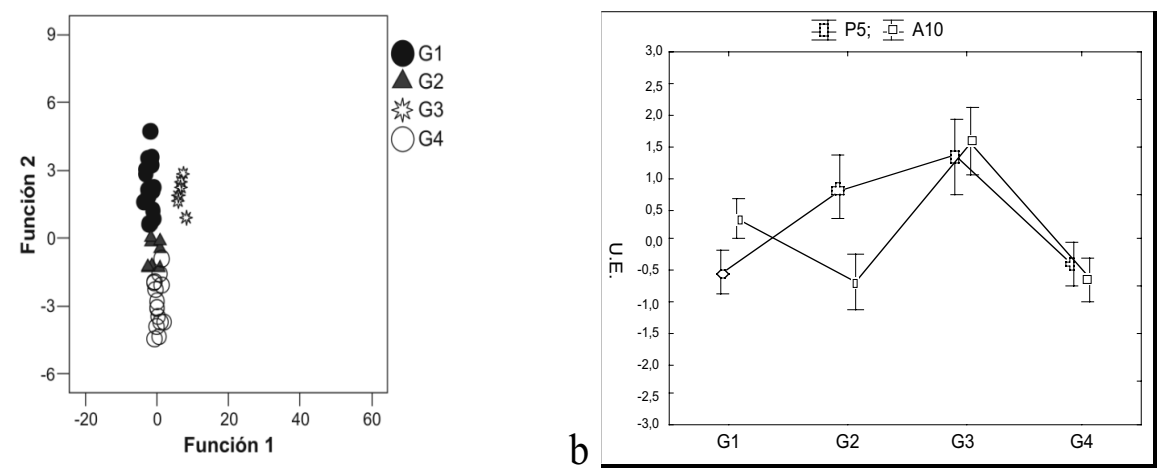

Figura 4. Representaciones gráficas de la CDA para el agrupamiento que resultó ser más discriminante (a), y de los valores medios de las variables más discriminantes en relación a este CDA -P5 y A10(b) -U.E., unidades estructurales-. CDA graphic representation for the most discriminant classification (a), and the average plot for the most discriminant variables $-P 5$ and A10- (b) -U.E., structural units-.

\section{RESULTADOS}

Los resultados obtenidos a partir de las matrices deducidas a partir de las georreferenciaciones elaboradas sobre las caracterizaciones de Franco (1994) y Costa et al. (1998), permitieron obtener un cluster, en cada caso, sobre los valores medios de presencia por taxon por área fitoclimática. Estos dendrogramas están expuestos en la figura 2.

El análisis de semejanzas, a partir de la caracterización de Franco (op. cit.), deja ver una distancia muy reducida entre las áreas fitoclimáticas de Noroeste Occidental, Terra Quente y Nordeste Leonés. Conviene aquí llamar la atención para el hecho de que esta caracterización considera al valle del río Támega como formando todavía parte del Noroeste Occidental, permitiendo así una mayor semejanza florística en relación al área que circunscribe como Terra Quente, recorrida por el río Duero antes de entrar en la zona de influencia atlántica. En relación al análisis realizados a partir de la caracterización de Costa et al. (op. cit.), resulta evidente la similitud obtenida entre el superdistrito Mínense-litoral y el subsector Margato-Sanabriense, atravesado por el río Támega antes de adentrarse en el primero de estos superdistritos; al mismo tiempo, se puede observar una semejanza relativamente menor, aunque todavía apreciable, en relación al superdistrito duriense, fronterizo con el Minense-litoral para el río Duero.

El análisis de semejanzas, a partir de la matriz de correlación Pearson calculada para la matriz numérica padronizada resultante de la georreferenciación con base en los mapas del IA portugués, está expuesto gráficamente en el dendrograma de la figura 3.

Los resultados del CDA para cada conjunto de agrupamientos deducidos a partir de las líneas fenogramáticas sobre el dendrograma anterior, además de las variaciones de los valores medios para las tres primeras variables más discriminantes, están ambos expuestos en la figura 4 (a y b, respectivamente).

Los valores numéricos de este análisis, por cada conjunto de agrupamientos, están recogidos en la tab. 2, indicando aquí el grado de discriminación por variable 
a

\begin{tabular}{|c|c|c|c|c|}
\hline Variable & Wilks' Lambda & $\mathbf{F}$ & df1 & Significancia \\
\hline A 10 & 0,40094 & 20,41988 & 3 & $2,98 \mathrm{E}-08$ \\
\hline P5 & 0,19335 & 16,98914 & 6 & $1,50 \mathrm{E}-12$ \\
\hline $\mathrm{t} 2$ & 0,389468649 & 32,91962 & 2 & $2,51 \mathrm{E}-09$ \\
\hline A4 & 0,319228975 & 15,78296 & 4 & $1,29 \mathrm{E}-09$ \\
\hline
\end{tabular}

Tabla 2. Valores numéricos asociados al CDA sobre la matriz numérica básica dividida en los cuatro grupos más discriminantes (a); y los referentes a las tres tendencias de comportamiento (b). Numerical values for the CDA upon the numerical matrix divided into four discriminate groups (a); and regarding to the three behaviour tendencies.

ambiental (Lambda de Willks y el valor de F retirado de cada etapa discriminante forward stepwise-).

A partir de los cuatro grupos obtenidos se procede a un segundo análisis, sobre cada uno de ellos. Los resultados por grupo están representados gráficamente en la figura 5 (a, b, c, d y e).

La variación media de la precipitación, temperatura, evapotranspiración y altitud determina el orden de los subgrupos, tal y como se expone en la figura 6a. Además de esta representación gráfica, se pueden observar igualmente las representaciones referentes a las amplitudes de cada uno de los parámetros bioclimáticos mencionados por subgrupo (fig. $6 \mathrm{~b}, \mathrm{c}, \mathrm{d}, \mathrm{e}$ ).

Con el objetivo de describir el comportamiento termopluviométrico y altitudinal que determina la existencia de los 17 subgrupos bioclimáticos obtenidos, se procede a un análisis de cluster sobre los valores medios por parámetro estudiado, para cada uno de los subgrupos mencionados. El dendrograma resultante está expuesto en la figura 7.

Aplicando nuevamente el protocolo metodológico propuesto, en este caso sobre el dendrograma anterior, se deducen un total de 3 tendencias, cuya CDA está representada en la figura $8 \mathrm{~b}$, estando los valores numéricos en la tab. 2b. En este caso, las variables ambientales más discriminantes son referentes a la temperatura y a la altitud, que de hecho son los que acaban por tener amplitudes de variación mayores (fig. 8a, d, f), en contraste con la precipitación y evapotranspiración, con variaciones menores (fig. 8a, c, e).

La distribución de cada taxon por tendencia general de comportamiento está enumerada en la tab. 3. Finalmente, a partir de la distribución de cada uno de los táxones que forma parte de los respectivos subagrupamientos, se exponen en la figura 9 las distribuciones relativas a cada uno de ellos para el área de estudio.

\section{DISCUSIÓN Y CONCLUSIONES}

Teniendo en consideración este protocolo metodológico, los resultados de la correlación entre la flora estudiada y los mapas bioclimáticos del IA portugués permitieron detectar un total de 17 subgrupos bioclimáticos. Para ello fueron seleccionados nuevos táxones, incidiendo en el uso de aquellos endémicos o subendémicos con presencia preponderante a partir de los 500 $\mathrm{m}$, con el objetivo de poder trabajar con individuos de distribución más restringida y, al mismo tiempo, más concentrada en las barreras montañosas (determinantes en la 

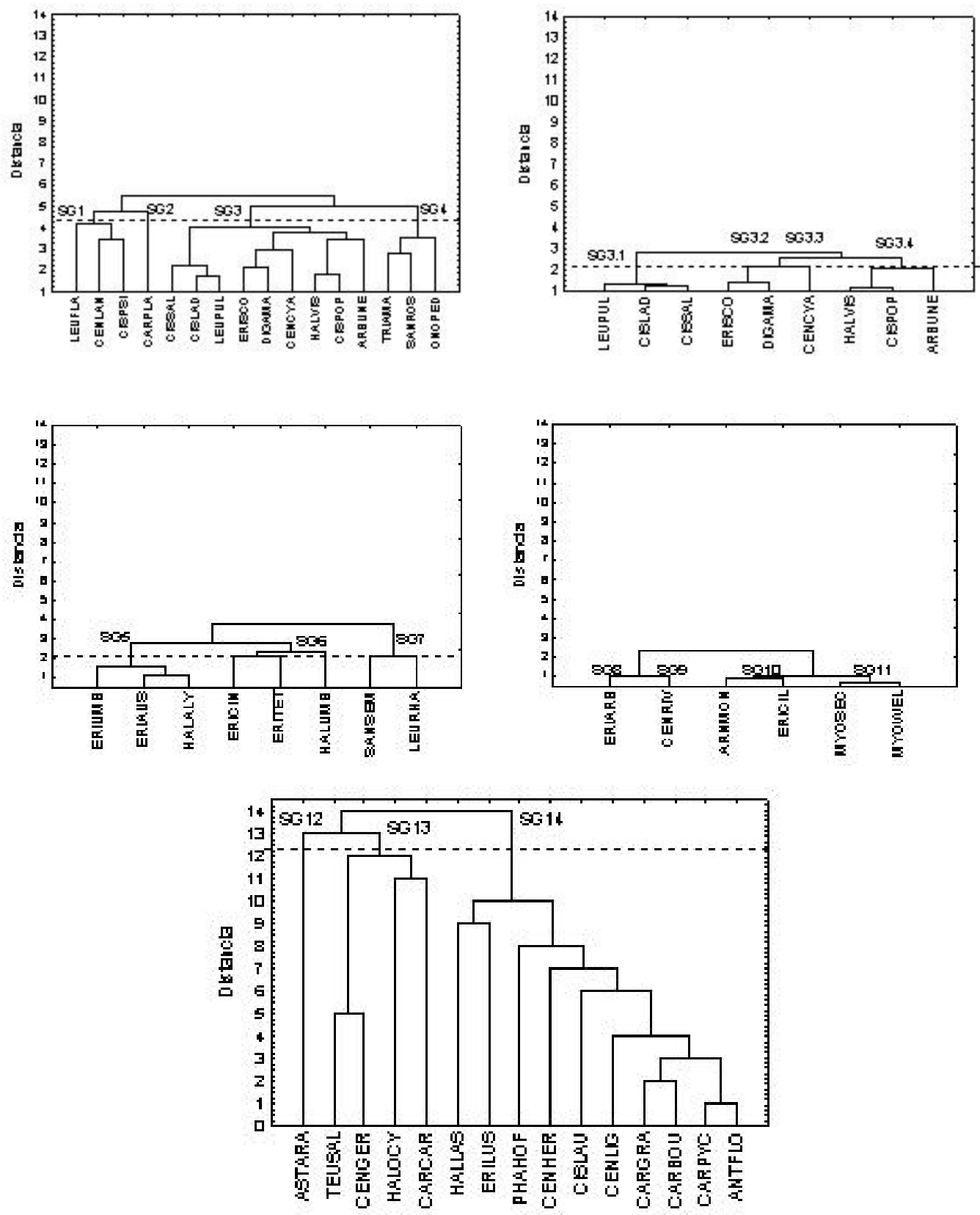

Figura 5. Representación en un dendrograma realizada a partir de la matriz de correlación del primer grupo (a) -G1-, del subgrupo 3 (SG3) de esta primera división (b); del segundo grupo (c) -G2-; del tercer grupo (d) -G3-; y del cuarto grupo (e) -G4-. Dendrogramatic representation obtained from the correlation for the first group (a) -G1, from its third subgroup (SG3) (b), for the third group (d) -G3, and for the fourth group (e) -G4-. 

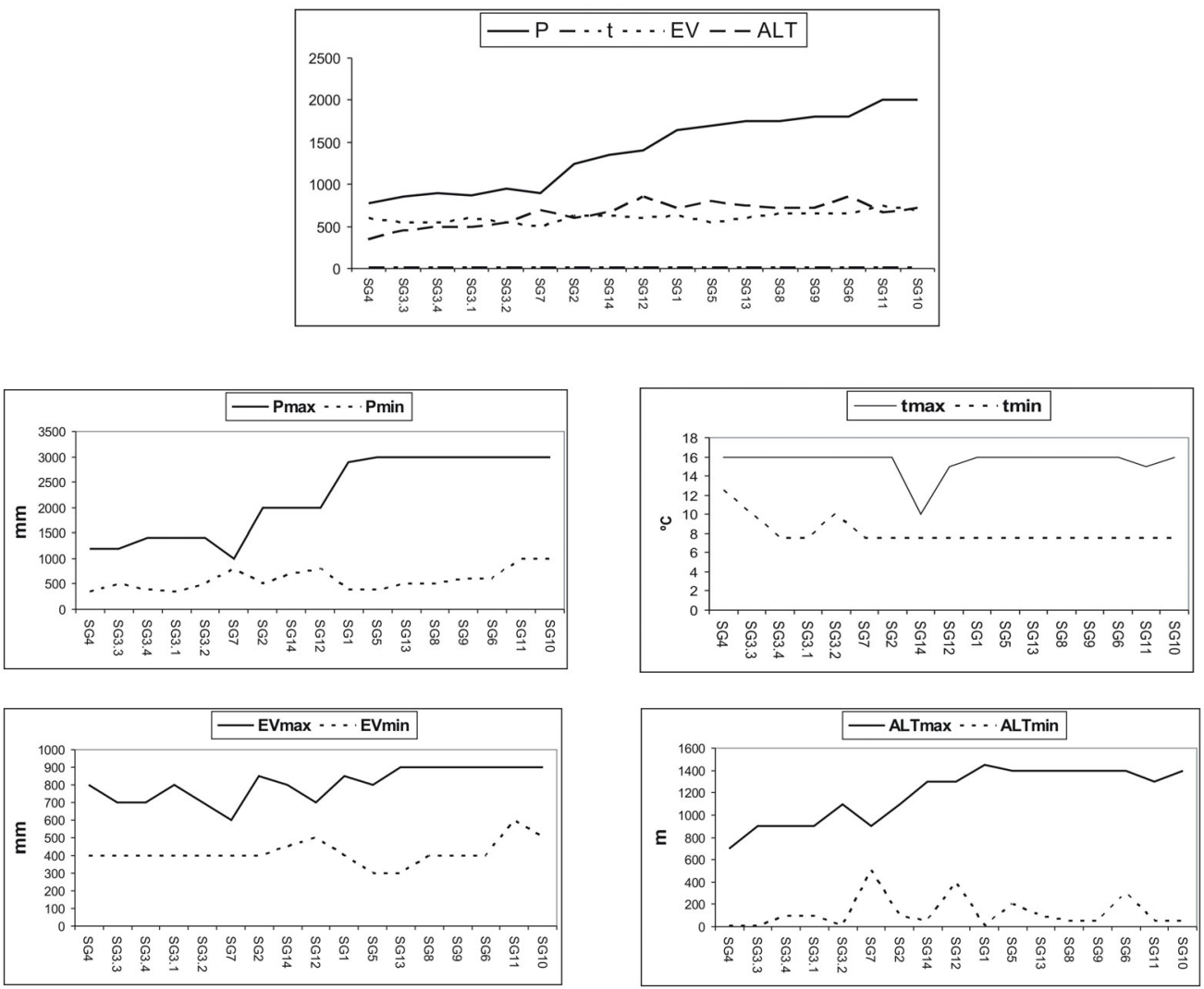

Figura 6. Representaciones gráficas de la variación media (a) y de las amplitudes por variable ambiental (b-e) (P, precipitación media; Pmax, precipitación media máxima; Pmin, precipitación media mínima; $\mathrm{t}$, temperatura media; tmax, temperatura media máxima; tmin, temperatura media mínima; EV, evapotranspiración media; EVmax, evapotranspiración media máxima; EVmin, evapotranspiración media mínima; ALT, altitud media; ALT max, altitud media máxima; ALT min, altitud media mínima). Average (a) and interval plots per environmental variable (b-e) (P, average precipitation; Pmax, highest average precipitation; Pmin, lowest average precipitation; $t$, average temperature; tmax, highest average temperature; tmin, lowest average temperature; EV, average evapotranspiration; EVmax, highest average evapotranspiration; EVmin, lowest average evapotranspiration; ALT, average altitude; ALT max, highest average altitude; ALT min, lowest average altitude).

diferenciación climática de esta parte del país). No obstante, los resultados aquí obtenidos no contrastan con los procedentes de las últimas contribuciones sobre correlación florístico-bioclimática (Crespí et al., 2001; Martins et al., 2004, 2006), donde no resulta tan evidente una diferenciación entre el dominio mediterráneo y el atlántico. Estos subgrupos forman parte de tres grandes tendencias de comportamiento, de acuerdo con las variables ambientales analizadas. En este sentido, resulta evidente observar como cada tendencia general de comportamiento responde a una progresión desde los valores ambientales más bajos (en la tendencia 1), hasta los más altos (en la tendencia 3). Una progresión así proporciona una idea de transformación gradual de las condiciones 


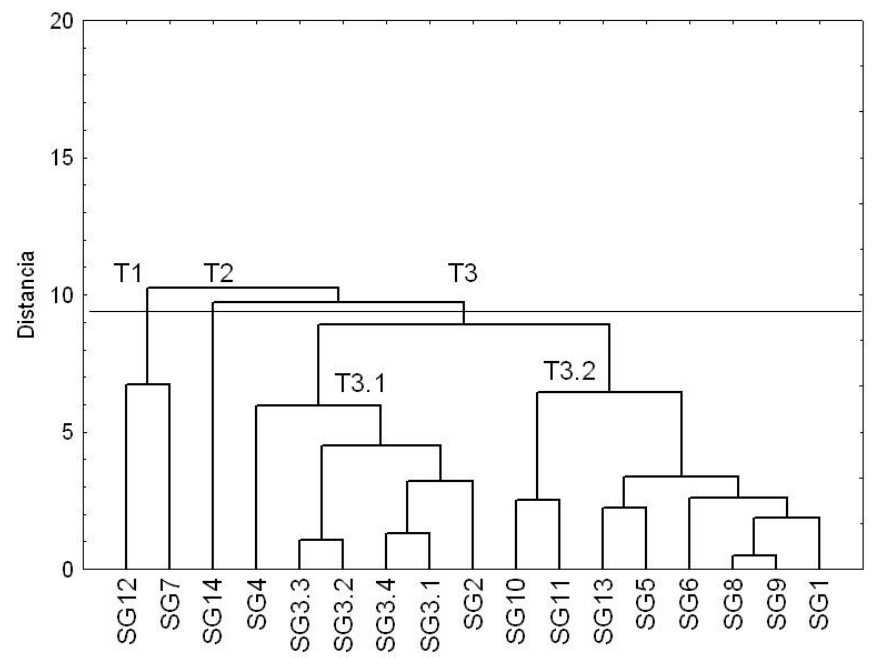

Figura 7. Dendrograma de los valores medios por parámetro ambiental estudiado, para cada uno de los subgrupos bioclimáticos obtenidos. Dendrogram of the average values per environmental parameter, for each bioclimatic subgroups obtained.

climáticas, incidiendo aquí de modo especial la temperatura y la altitud, con variaciones más marcadas para la primera y la tercera tendencia. Al mismo tiempo, el hecho de encontrar tres tendencias bioclimáticas más discriminantes lleva a pensar en la posibilidad de no ser tan acentuado el tránsito atlántico-mediterráneo, contrariamente a lo que ha venido siendo apuntado por diversos autores, a partir del estudio de la vegetación como referencia bioclimática (Alburquerque, 1941, 1943; Rozeira, 1944; Azevedo, 1953a, b; Gonçalves, 1985; Ribeiro, 1991; Molina et al., 1992; Rivas-Martínez 1987; Franco 1994; Correia, 1997; Costa et al., 1998; Lousã, 2004; González, 2005). No obstante, estas diferencias son también el resultado de distinciones claras entre los tratamientos metodológicos utilizados, una vez que la caracterización a través de unidades de vegetación permite el uso de unidades de variación más definidas (como es posible observar en la cartografía presentada por Capelo et al., 2007), al contrario que las unidades florísticas. En estas últimas, la propia distribución del taxon constituye un elemento de variabilidad mucho más indefinido, pues sus intervalos ecológicos permiten frecuentemente su presencia en diferentes unidades de vegetación. El principal resultado de esta distinción entre unidades de variación empleadas, acaba por ser determinante a la hora de analizar la transición atlántico-mediterránea. Observando esas distribuciones no es posible concluir cual de ellas formaría parte de un comportamiento intermedio, una vez que aunque se verifique una ligera concentración al este de la T1, la T2 y la T3 acaban por abarcar transversalmente todo el área de estudio. Una visión más detallada de la T2 permite observar un ejemplo característico de ambientes húmedos y frescos (como se puede deducir atendiendo a su restringida variación para los parámetros ambientales considerados), extendiéndose desde la zona atlántica hasta la mediterránea, buscando en altitud precipitaciones más altas y temperaturas más suaves en los períodos estivales. Por su parte, la T1, al contrario que 

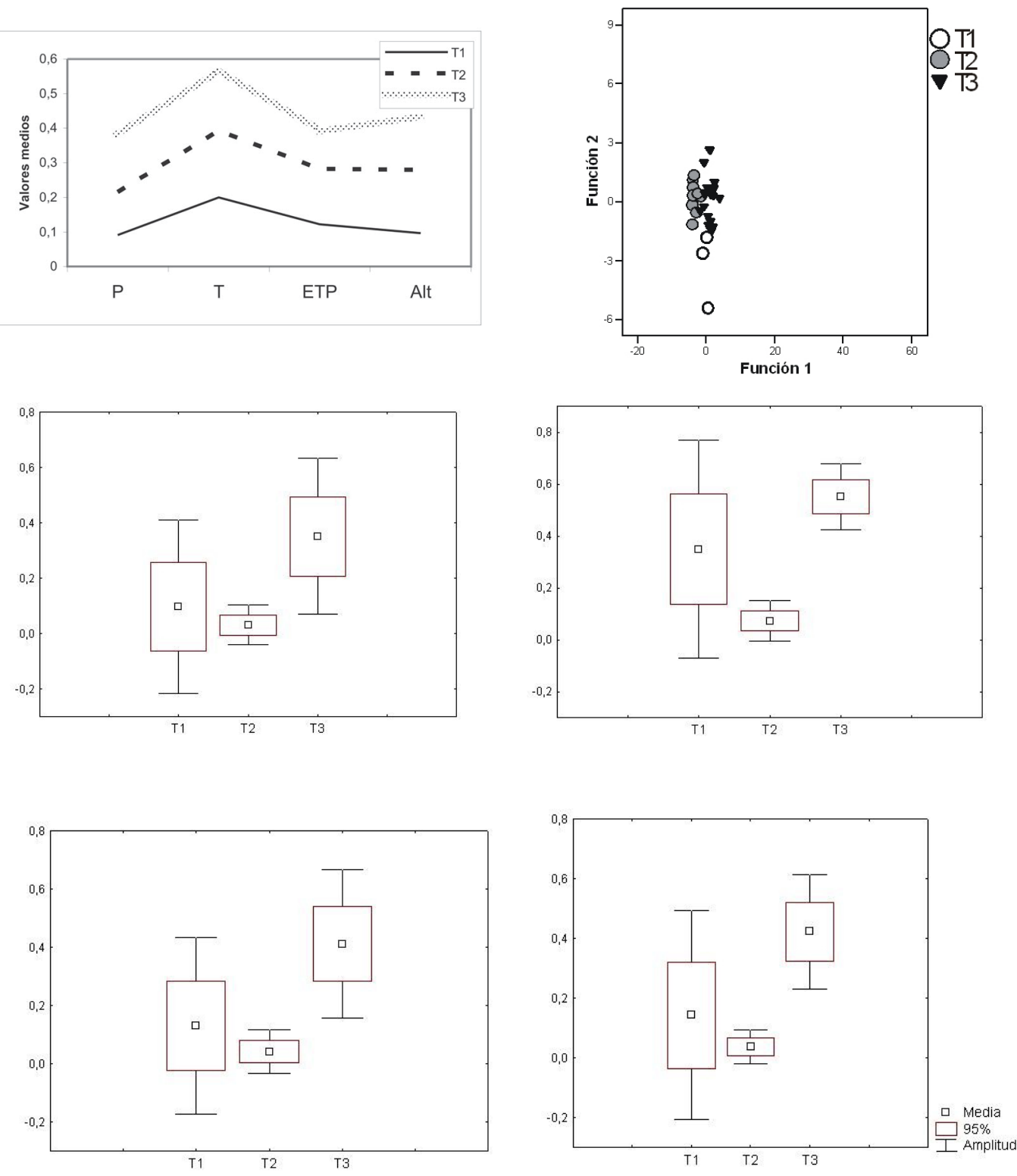

Figura 8. Evolución media de los parámetros ambientales por tendencia de comportamiento (a); representación de la CDA sobre la matriz florístico-ambiental, organizada por tendencia de comportamiento (b); variabilidad por tendencia para la precipitación (c); variabilidad por tendencia para la temperatura (d); variabilidad por tendencia para la evapotranspiración (e); variabilidad por tendencia para la altitud (f). Average plot of the environmental parameters per behaviour tendency (a); CDA representation for the floristic-environmental matrix per behaviour tendency (b); variability of precipitation per tendency (c); variability of temperature per tendency (d); variability of evapotranspiration per tendency (e); variability of altitude per tendency $(f)$. 


\begin{tabular}{|c|c|c|c|c|c|}
\hline Tendencia & Taxon & Tendencia & Taxon & Tendencia & Taxon \\
\hline $\mathrm{T} 1$ & $\begin{array}{c}\text { Leuzea } \\
\text { rhaponticoides } \\
\text { Graells }\end{array}$ & $\mathrm{T} 3(3.2)$ & $\begin{array}{c}\text { Halimium } \\
\text { lasianthum subsp } \\
\text { alyssoides (Lam.) } \\
\text { Greuter }\end{array}$ & $\mathrm{T} 3(3.2)$ & $\begin{array}{c}\text { Cistus psilosepalus } \\
\text { Sweet }\end{array}$ \\
\hline $\mathrm{T} 1$ & $\begin{array}{c}\text { Santolina } \\
\text { semidentata Hoff \& } \\
\text { Liink }\end{array}$ & $\mathrm{T} 3(3.2)$ & Erica cinerea $\mathrm{L}$. & $\mathrm{T} 3(3.2)$ & $\begin{array}{l}\text { Leucanthemopsis } \\
\text { pallida (Miller) } \\
\text { Heywood subsp. } \\
\text { flaveola } \\
\text { (Hoffmanns. \& } \\
\text { Link) Ladero \& } \\
\text { Velasco }\end{array}$ \\
\hline $\mathrm{T} 1$ & $\begin{array}{c}\text { Aster aragonensis } \\
\text { Asso }\end{array}$ & $\mathrm{T} 3(3.2)$ & Erica tetralix $\mathrm{L}$. & $\mathrm{T} 3(3.1)$ & $\begin{array}{l}\text { Carduus platypus } \\
\text { Lange subsp. } \\
\text { platypus }\end{array}$ \\
\hline $\mathrm{T} 2$ & $\begin{array}{l}\text { Anthemis triumfetti } \\
\text { (L.) DC. f. flosculosa } \\
\text { (Briq. \& Cavill.) R. } \\
\text { Fernandes }\end{array}$ & $\mathrm{T} 3(3.2)$ & $\begin{array}{l}\text { Halimium } \\
\text { umbellatum }(\mathrm{L} .) \\
\text { Spach subsp } \\
\text { umbellatum }\end{array}$ & $\mathrm{T} 3(3.1)$ & Arbutus unedo L \\
\hline $\mathrm{T} 2$ & $\begin{array}{l}\text { Carduus platypus var. } \\
\text { gramatensis (Wk.) P. } \\
\text { Coutinho }\end{array}$ & $\mathrm{T} 3(3.2)$ & Erica arborea $\mathrm{L}$. & $\mathrm{T} 3(3.1)$ & $\begin{array}{c}\text { Cistus populifolius } \\
\text { L. }\end{array}$ \\
\hline $\mathrm{T} 2$ & $\begin{array}{l}\text { Carduus bourgeanus } \\
\text { Boiss \& Reuter }\end{array}$ & $\mathrm{T} 3(3.2)$ & $\begin{array}{c}\text { Centaurea rivularis } \\
\text { Brot. }\end{array}$ & $\mathrm{T} 3(3.1)$ & $\begin{array}{c}\text { Halimium } \\
\text { umbellatum (L.) } \\
\text { Spach subsp. } \\
\text { viscosum (Wk.) } \\
\text { O.Bolòs \& Vigo }\end{array}$ \\
\hline $\mathrm{T} 2$ & $\begin{array}{c}\text { Carduus } \\
\text { pycnocephalus } \mathrm{L} .\end{array}$ & $\mathrm{T} 3(3.2)$ & $\begin{array}{l}\text { Arnica montana L. } \\
\text { subsp. atlantica A. } \\
\text { Bolós }\end{array}$ & $\mathrm{T} 3(3.1)$ & Cistus ladanifer L. \\
\hline $\mathrm{T} 2$ & $\begin{array}{l}\text { Centaurea herminii } \\
\text { Rouy subsp. herminii }\end{array}$ & $\mathrm{T} 3(3.2)$ & Erica ciliaris $\mathrm{L}$. & $\mathrm{T} 3(3.1)$ & Cistus salvifolius L. \\
\hline $\mathrm{T} 2$ & $\begin{array}{l}\text { Centaurea triumfetti } \\
\text { All. subsp. lingulata } \\
\text { (Lag.) Vicioso }\end{array}$ & $\mathrm{T} 3(3.2)$ & $\begin{array}{c}\text { Myosotis secunda } \mathrm{A} . \\
\text { Murray }\end{array}$ & $\mathrm{T} 3(3.1)$ & $\begin{array}{c}\text { Leucanthemopsis } \\
\text { pallida (Miller) } \\
\text { Heywood subsp. } \\
\text { pulverulenta (Lag.) } \\
\text { Bolós et Vigo }\end{array}$ \\
\hline $\mathrm{T} 2$ & Cistus laurifolius L. & $\mathrm{T} 3(3.2)$ & $\begin{array}{l}\text { Myosotis welwistchi } \\
\text { Boiss. et Reut. }\end{array}$ & $\mathrm{T} 3(3.1)$ & $\begin{array}{c}\text { Digitalis amandiana } \\
\text { Samp. }\end{array}$ \\
\hline $\mathrm{T} 2$ & $\begin{array}{c}\text { Erica lusitanica } \\
\text { Rodolphi }\end{array}$ & $\mathrm{T} 3(3.2)$ & $\begin{array}{l}\text { Carduus carpetanus } \\
\text { Boiss. et Reuter }\end{array}$ & $\mathrm{T} 3(3.1)$ & Erica scoparia $\mathrm{L}$. \\
\hline $\mathrm{T} 2$ & $\begin{array}{l}\text { Halimium lasianthum } \\
\text { subsp lasianthum }\end{array}$ & $\mathrm{T} 3(3.2)$ & $\begin{array}{c}\text { Centaurea } \\
\text { geresensis J. Arènes }\end{array}$ & $\mathrm{T} 3(3.1)$ & Centaurea cyanus $\mathrm{L}$. \\
\hline $\mathrm{T} 2$ & $\begin{array}{l}\text { Phalacrocarpum } \\
\text { hoffmannseggii } \\
\text { (Samp.) Laínz }\end{array}$ & $\mathrm{T} 3(3.2)$ & $\begin{array}{l}\text { Halimium ocymoides } \\
\text { (Lam.) Wk. }\end{array}$ & $\mathrm{T} 3(3.1)$ & $\begin{array}{c}\text { Ononis viscosa ssp } \\
\text { pedroi Crespí, } \\
\text { Castro et Bernardos }\end{array}$ \\
\hline $\mathrm{T} 3(3.2)$ & Erica australis L. & $\mathrm{T} 3(3.2)$ & $\begin{array}{c}\text { Teucrium } \\
\text { salviastrum } \text { Schreb. }\end{array}$ & $\mathrm{T} 3(3.1)$ & $\begin{array}{c}\text { Santolina } \\
\text { rosmariinifolia L. }\end{array}$ \\
\hline $\mathrm{T} 3(3.2)$ & Erica umbellata L. & $\mathrm{T} 3(3.2)$ & $\begin{array}{c}\text { Centaurea laingeana } \\
\text { Wk. }\end{array}$ & $\mathrm{T} 3(3.1)$ & $\begin{array}{c}\text { Trigonella } \\
\text { amandiana Samp. }\end{array}$ \\
\hline
\end{tabular}

Tabla 3. Relación de taxa ordenados por tendencia de comportamiento y por subtendencia, en el caso de la T3 (3.1 y 3.2). List of taxa ordered by behaviour tendency and subtendency, in the case of T3 (3.1 and 3.2). 
la tendencia anteriormente mencionada, es típicamente continental, atendiendo a los valores medios de temperatura y de precipitación más altos y más variables que los anteriores, lo que acaba por proporcionar una idea de intervalos termopluviométricos más acentuados. Finalmente, la T3, formada por casi un $70 \%$ de los táxones estudiados, es la tendencia que mejor deja ver el efecto gradual atlántico-mediterráneo, tal y como se deduce de las dos subtendencias que forman parte de ésta. Subgrupos como el 3.1, el 5 y el 6 demuestran una distribución más concentrada en el centro y este del área estudiada, aunque eso no impide que parte de esta distribución se prolongue hasta la mitad atlántica, utilizando para eso el corredor establecido por el río Duero. Por su lado, la subtendencia 3.2 deja que táxones con su óptimo de distribución en la mitad atlántica, puedan extender su presencia a lo largo de cotas más elevadas de la mitad mediterránea, incidiendo nuevamente en el aspecto de transición bioclimática gradual. De este modo, y como confirmación de ese fenómeno progresivo de transición atlánticomediterránea, una evolución ambiental como ésta no ofrece una discriminación significativa, pasando a formar parte de un proceso con acusadas sobreposiciones, tal y como se observa claramente en las cartografías de cada una de las dos subtendencias detectadas. El evidente fenómeno aislante de la cadena montañosa Larouco-Alvão-Marão-Montemuro-LeomilLapa, que se extiende desde el centro-norte hasta el sureste, en conjunción con el corredor transversal formado por el río Duero y su afluente, el río Tâmega, constituyen, aparentemente, un efecto combinado determinante para diluir la transición atlántico-mediterránea. Esta combinación provoca un prolongamiento de la florística Galaico-Portuguesa sobre la Lusitano-Duriense; al mismo tiempo que facilita la posibilidad de alargar esas distribuciones florísticas por el supramediterráneo Salmantino-Duriense.

Tal y como afirma Gavilán \& Fernández-González (1997) y Gavilán et al. (1998), la correlación entre precipitación y temperatura con la altitud podría ser, en este caso, una razón de peso para incidir en ese carácter gradual de la variación climática entre la influencia atlántica y la mediterránea, combinado con el efecto transversal provocado por los cursos fluviales del Duero y del Támega. De hecho, también Molina et al. (1992) detectaron discriminaciones más acentuadas entre el piso supramediterráneo y los restantes para Portugal, pues las diferencias entre el mesomediterráneo y el termomediterráneo eran claramente menores. Al mismo tiempo, los resultados obtenidos para estos táxones con las caracterizaciones de Franco (1994) y de Costa et al. (1998), dejan ver como la influencia atlántica (Noroeste Occidental, según Franco, o superdistrito Mínenselitoral, según Costa et al.) presenta una estrecha relación con aquellas áreas que son inmediatamente atravesadas por los dos ríos mencionados: En este sentido, los resultados obtenidos apuntan a una banda de transición atlántico-mediterránea, que se extiende por el lado este de la cadena montañosa GerêsMontemuro-Leomil-Lapa. Esta área, hasta ahora designada como noroeste montano (según Franco, 1994), o como subsector Geresiano-Queixense, superdistrito AlvãoMarão, superdistrito beira-duriense y superdistrito Altibeirense (Costa et al., 1998), daría paso a una transición fitoclimática, como resultado de la influencia atlántica que penetra por los ríos Duero y Támega. Al mismo tiempo, esa cordillera montañosa no ofrece características homogéneas. Tal y como afirma Costa et al. (op. cit.), es posible diferenciar una parte más fría y húmeda, al norte del Duero, de otra 

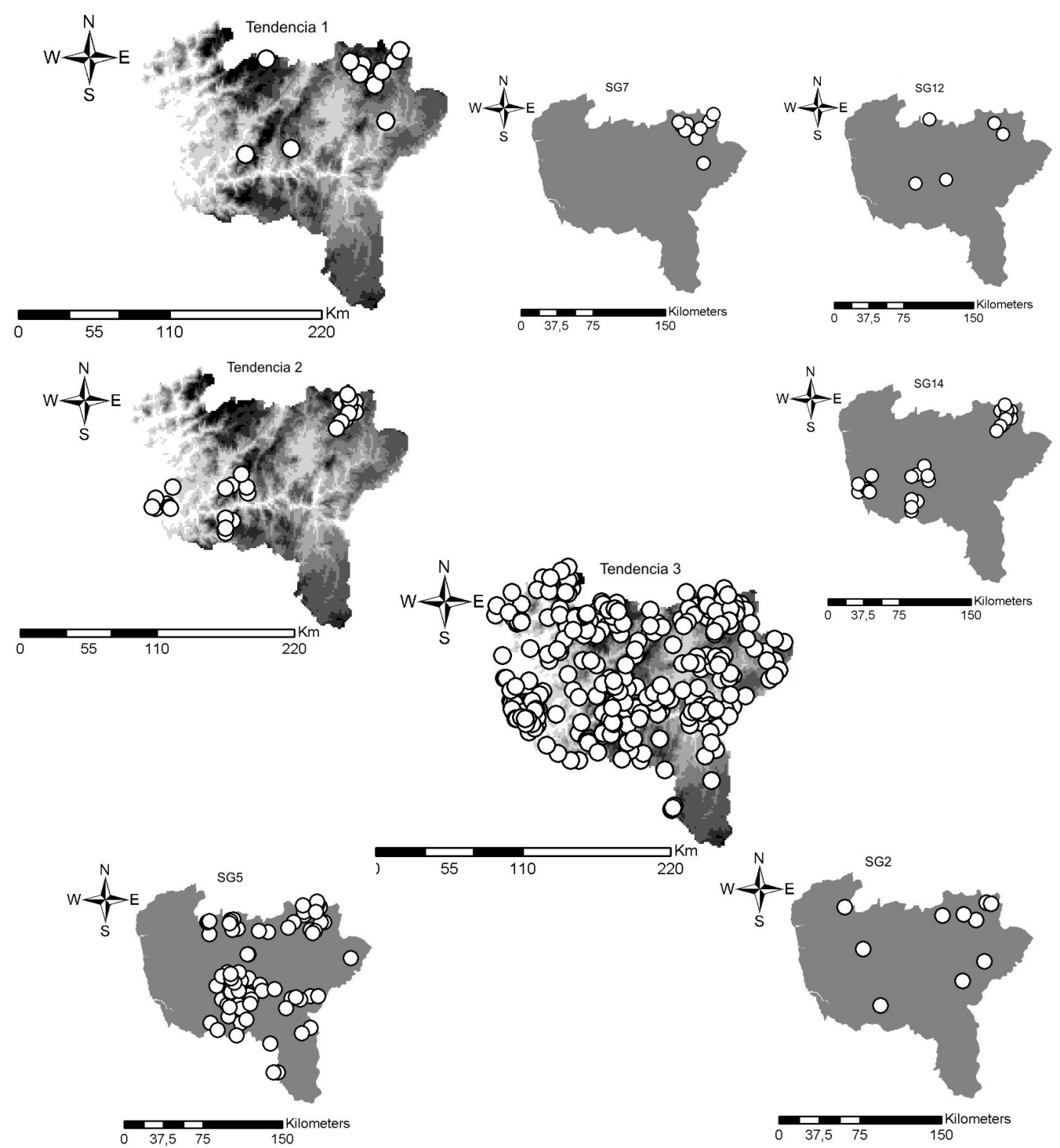

$\begin{array}{llll} & & & \\ 0 & 37,5 & 75 & 150\end{array}$
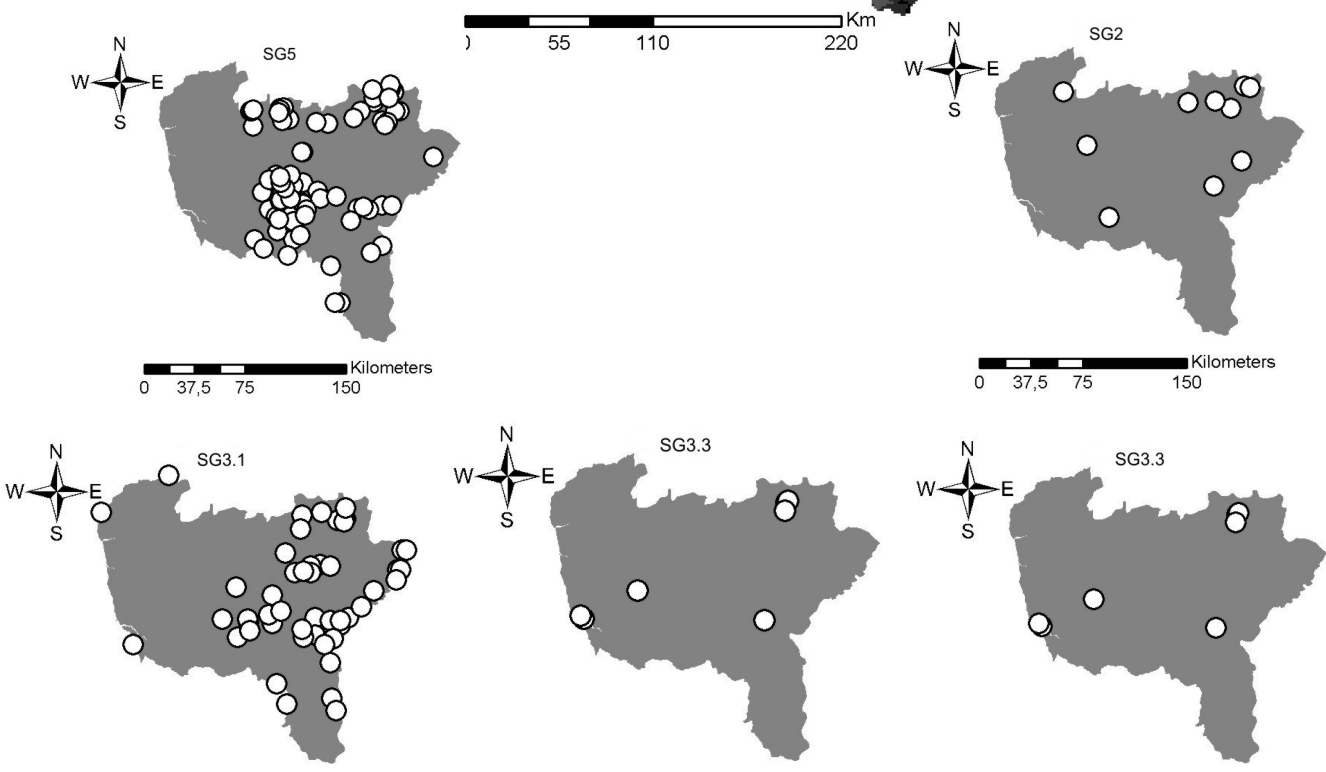

\begin{tabular}{llll}
\hline & \multicolumn{1}{c}{ Kilometers } & & \\
0 & 37,5 & 75 & 150
\end{tabular}
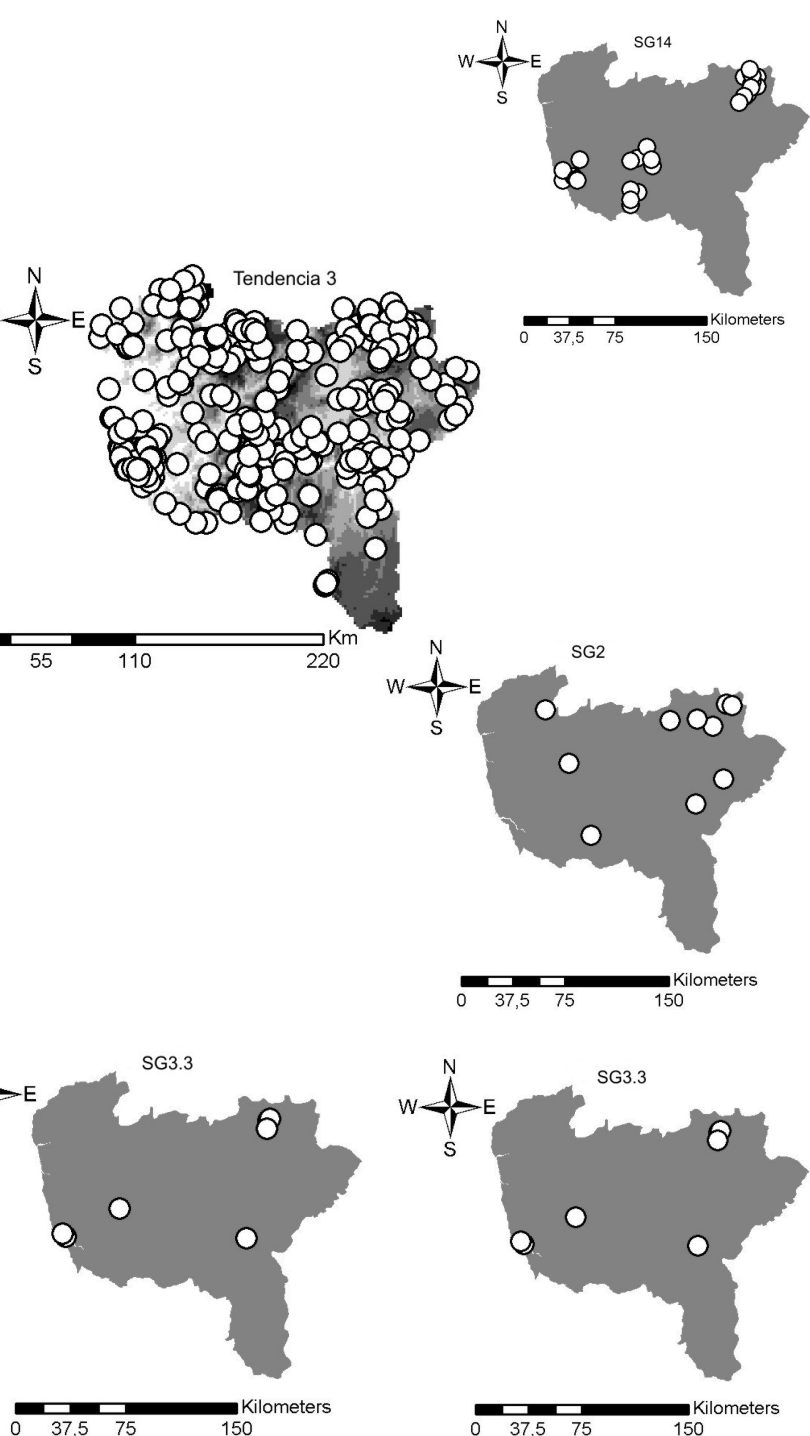

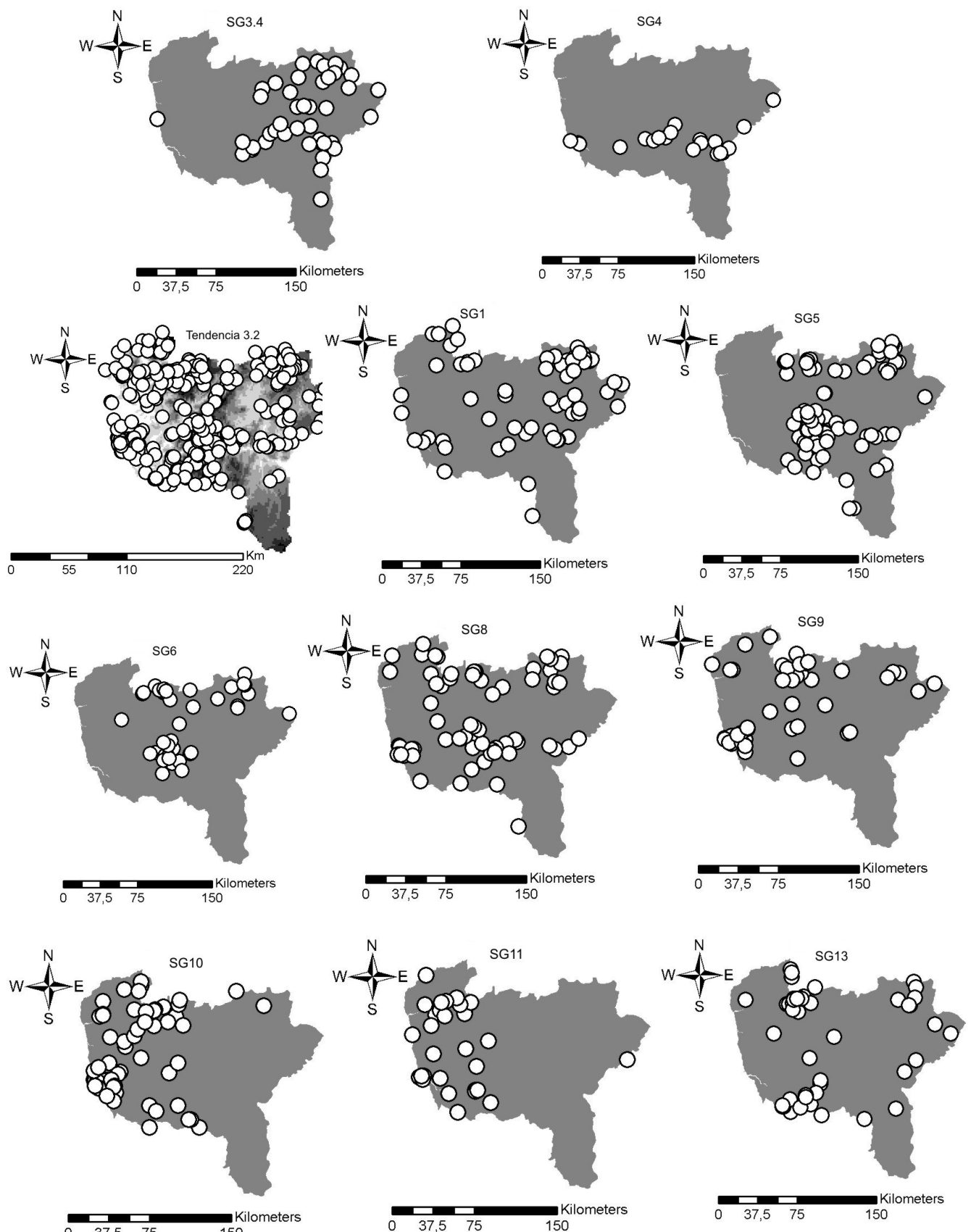

Figura 9. Distribuciones de los táxones que forman parte de cada uno de los subgrupos deducidos, para el área de estudio (SG1-14), organizados por tendencias generales de comportamiento (T1-3) y de las dos subtendencias de la T3 (T3.1 y T3.2). Occurrence of taxa per subgroup, for the study area (SG1-14), classified per behaviour tendencies (T1-3) and the subtendencies of the T3 (T3.1 and T3.2). 
más cálida y seca, al sur del mismo río. Por su parte, la vertiente norte también presenta una transición climática, comprendida entre el río Támega y el Duero. Tal circunstancia, que continuará siendo objeto de posteriores análisis en próximas contribuciones, incidirá en la dificultad de delimitar una frontera clara atlántico-mediterránea, al menos, para el Norte de Portugal.

AGRADECIMIENTOS. Los autores agradecen al proyecto POCI/BIA-BDE/56044/200 y a la Acción Integrada Luso-Española E-115/07 por la ayuda financiera prestada para la elaboración de este trabajo.

\section{BIBLIOGRAFIA}

ALBURQUERQUE, J. DE P. M. -1941- A Beira trasmontana na carta regional portuguesa. Agronomia Lusitana 3(3): 145-181.

ALBURQUERQUE, J. DE P. M. -1943- Zonagem climatológica. Como se tem feito em Portugal. Agronomia Lusitana 5(3): 191-225.

ALLÚE ANDRADE, J. L. -1990- Atlas fitoclimático de España. Taxonomias. Ed. Ministerio de Agricultura, Pesca y Alimentación (Instituto Nacional de Investigaciones Agrarias): 22-35. Madrid.

AZEVEDO, A. L. -1953a- O clima de Portugal. Contribuição para o estudo de alguns factores climáticos nas suas relações com a agricultura. Agros 36(3): 115-138.

AZEVEDO, A. L. -1953b- O clima de Portugal. Contribuição para o estudo de alguns factores climáticos nas suas relações com a agricultura. Agros 36(4-5): 157-201.

CAPELO, J., S. MESQUiTA, , J. C. COSTA, S. RIBEIRO, P. ARSÉNIO, C. NETO, T. MONTEIRO-HENRIQUES, C. AGUIAR, J. HONRADO, D. ESPÍRITO-SANTO \& M. LOUSÃ -2007-A mehodological approach to potential vegetation modeling using gis techniques and phytossociological expertknowledge: application to mainland portugal. Phytocoenologia 37 (3-4): 399-415.

CARBALLEIRA, A. -1985- Subregiones bioclimáticas de Galicia (clasificación y capacidades de cultivo de Actinidia chinensis Planch.). Actas das I Jornadas Técnicas sobre Actinidia Vigo.

CARBALleirA, A., C. DEVESA, R. RETUERTO, E. SANTILLAN \& F. UCIEDA -1983- Bioclimatología de Galicia. Ed. Fundación Barrié de la Maza Conde de Fenosa, Vigo.

CORREIA, A. I. -1997- Essai de phytoclimatologie dynamique sur le nort du Portugal. Lasgacalia, 19(1-2): 413-422.

COSTA, J. C., C. AGUIAR, CAPELO, J. H., M. LOUSÃ \& C. NETO -1998- Biogeografia de Portugal Continental. Quercetea 0: 5-56.

CRESPÍ, A. L., L. SILVA, , J. A. RIBEIRO, A. COELHO, F. AMICH \& S. BERNARDOS 2001- Modelo de caracterização fitoclimatica do Nordeste de Portugal. Análise metodológica e primeiros resultados. Silva Lusitana 9(1): 69-81.

CRESPÍ, A. L., A. CASTRO \& S. BERNARDOS -2005a- Flora da Região Demarcada do Douro, vol. 1-3, João Azevedo Editor. Mirandela.

CRESPÍ, A. L., A. MARTINS, A. CASTRO, C. AFONSO, S. BERNARDOS, A. GUIMARÃES \& F. AMICH -2005b-. Contribución al conocimiento de la flora del NE de Portugal. II. El género Trifolium L. Lazaroa 26: 41-53.

FRANCO, J. DE A. -1994- Zonas fitogeográficas predominantes de Portugal Continental. Annais Instituto Superior de Agronomia 44: 39-56.

GAVILÁN, R. G. \& F. FERNÁNDEZ-GONZÁLEZ -1997- Climatic discrimination of Mediterranean broad-leaved sclerophyllous and deciduous forest in central Spain. Journal of Vegetation Science 8: 377-386.

GAVILÁN, R. G., F. FERNÁNDEZ-GONZÁLEZ \& C. BLASI -1998- Climatic classification and ordination of the Spanish Sistema Central: relationships withs potential vegetation. Plant Ecology 139: 1-11.

GONZÁLEZ, S.del R. -2005- El cambio climático y su influencia en la vegetación de Castilla-León (España). Itinera Geobotanica 16: 5-533.

GONÇALVES, D. A. -1985- A rega de lima no 
interior de Trás-os-Montes. Instituto Universitário Politécnico de Trás-os-Montes e Alto Douro, Vila Real.

GOTELLI, N. J. \& A. M. ELLISON -2004- $A$ primer of ecological statistics. Sinauer Associates, Inc. Sunderland.

LOUSÃ, M. F. -2004- Bioclimatologia e séries de vegetação de Portugal. Lazaroa 85: 83-86.

MARTINS, A. R., A. L. CRESPÍ, S. BERNARDOS, M. A. BRANCO, A. CASTRO, C. P. FERNANDES, A. JANIAK, C. SANTOS, C. AFONSO, G. CARVALHO, A. LOBATO, A. HOELZER, F. AMICH \& B. WOZIWODA -2004- Sistema de caracterización fitoclimatológico de táxones en el Norte de Portugal. II. Grupos bioclimáticos. Boletín de la Real Sociedad Española de Historia Natural (Sec. Biologica) 99 (1-4), 1-5.

MARTINS. A., A. L. CRESPÍ, A. CASTRO, C. P. FERNANDES, J. ROCHA, S. BERNARDOS, C. AGUIR \& F. AMICH 2006- Contribución para la caracterización florístico-ambiental del Norte de Portugal.
Botanica Complutensis ( in press).

MOLINA, R. T., T. R. TELLEZ \& J. D. ALCARAZ -1992- Aportación a la bioclimatologia de Portugal. AnalesJjard. Bot. Madrid 49(2): 245-264.

RETUERTO, R. \& A. CARBALLEIRA -1991Definig phytoclimatic units in Galicia, Spain, by means of multivariate methods. Journal of Vegetation Science 2: 699-710.

RIBEIRO, O. -1991- Portugal. O Mediterrâneo e o Atlântico. Livraria Sá da Costa ed., col. Nova Universidade, Lisboa.

RIVAS-MARTÍNEZ, S. -1987-. Nociones sobre fitossociología, biogeografía y bioclimatología. In La vegetación de España, Peinado Lorca, M. \& Rivas-Martínez, S. ed., Colección Aula Abierta, Universidad de Alcalá de Henares. Alcalá de Henares.

ROZEIRA, A. -1944-. A flora da província de Trás-os-Montes e Alto Douro. Memórias da Sociedade. Broteriana, III: 110-118.

TABORDA, V. -1987-Alto Trás-os-Montes. Estudo geográfico. livros Horizonte ed., colecção. Espaço e sociedade, Lisboa. 
